\title{
Comment on 'Difference of uveal parameters between the acute primary angle closure eyes and the fellow eyes'
}

\author{
Kai Xiong Cheong ${ }^{1} \cdot$ Colin S. Tan $\mathbb{D}^{2,3}$
}

Received: 3 June 2018 / Accepted: 5 July 2018 / Published online: 31 July 2018

(C) The Royal College of Ophthalmologists 2018

In a study on acute primary angle closure, it was demonstrated that the anterior placement of the ciliary body and ciliary body thickness were positively correlated with choroidal thickness [1]. Knowledge of the effects of acute primary angle closure on the choroid is important because the choroid plays important roles in the eye. We would like to highlight aspects of the study design which may have bearing on the result interpretation.

The authors mentioned that the effects of diurnal variations were reduced by performing all examinations at about 1000 hours. There are studies that have used spectraldomain optical coherence tomography to demonstrate diurnal variation of choroidal thickness [2, 3]. The difference between the maximum and minimum choroidal thickness exceeded $30 \mu \mathrm{m}[2,3]$. In addition, a sub-analysis of subjects with thicker choroids (defined as $\geq 400 \mu \mathrm{m}$ ) in one paper [2], demonstrated that the mean amplitude was even larger $(43.1 \mu \mathrm{m})$ with a maximum of $59 \mu \mathrm{m}$. While considering diurnal variation is an important consideration, a consequence of this is that the choroidal thickness measurements would be made at different times from the initial APAC attack. This is likely to influence the correlation of choroidal thickness with the anterior placement of the ciliary body and ciliary body thickness. However, if diurnal variation is to be considered, this is likely a limitation which cannot be overcome.

Another important consideration is the topographic variation of the choroid. The choroid is a complex, three-

Colin S. Tan

Colintan_eye@yahoo.com.sg

1 Singapore National Eye Centre, Singapore Health Services, Singapore, Singapore

2 Department of Ophthalmology, Tan Tock Seng Hospital, Singapore, Singapore

3 Fundus Image Reading Center, National Healthcare Group Eye Institute, Singapore, Singapore dimensional structure comprising an anastomosing blood vessel network. Studies have reported significant variation of choroidal thicknesses at different regions of the macula using the Early Treatment Diabetic Retinopathy Study (ETDRS) grid $[4,5]$. In this study, the mean overall regional thicknesses of the choroid in the 36 sectors of the sweptsource grid were calculated. However, because this is different from the choroidal thicknesses reported in earlier studies, comparison of the current study with similar studies is difficult. It would be interesting to determine if anterior placement of the ciliary body and ciliary body thickness are still positively correlated with choroidal thickness at the various ETDRS sectors. This would facilitate comparison with other studies.

In conclusion, we congratulate the authors on an interesting paper.

Funding Dr Cheong does any receiving funding. Dr Tan receives research funding from the National Medical Research Council Transition Award (Code: NMRC/TA/0039/2015). Dr Tan also receives conference support from Bayer (South East Asia) Pte. Ltd., Heidelberg Engineering (Heidelberg, Germany), and Novartis (Singapore).

\section{Compliance with ethical standards}

Conflict of interest The authors declare that they have no conflict of interest.

\section{References}

1. Li X, Wang W, Huang W, et al. Difference of uveal parameters between the acute primary angle closure eyes and the fellow eyes. Eye. 2018.

2. Tan CS, Ouyang Y, Ruiz H, Sadda SR. Diurnal variation of choroidal thickness in normal, healthy subjects measured by spectral domain optical coherence tomography. Invest Ophthalmol Vis Sci. 2012;53:261-6.

3. Usui S, Ikuno Y, Akiba M, et al. Circadian changes in subfoveal choroidal thickness and the relationship with circulatory factors in healthy subjects. Invest Ophthalmol Vis Sci. 2012;53:2300-7. 
4. Tan CS, Cheong KX, Lim LW, Li KZ. Topographic variation of choroidal and retinal thicknesses at the macula in healthy adults. $\mathrm{Br}$ J Ophthalmol. 2013;98:339-44.
5. Tan CS, Cheong KX. Macular choroidal thicknesses in healthy adults-relationship with ocular and demographic factors. Invest Ophthalmol Vis Sci. 2014;55:6452-8.

\title{
Comment on: Difference of uveal parameters between the acute primary angle closure eyes and the fellow eyes
}

\author{
Xingyi $\mathrm{Li}^{1} \cdot$ Wei Wang ${ }^{1} \cdot$ Xiulan Zhang $\mathbb{1}^{1}$
}

Received: 15 June 2018 / Accepted: 5 July 2018 / Published online: 31 July 2018

(c) The Royal College of Ophthalmologists 2018

We read with interest the comment by Cheong et al. [1].

As the comment mentioned, in our study, the diurnal variations was an insurmountable confounding factor to the thickness of choroid and other parts of uvea. As we know, APAC attack usually happened at night, which was speculated be associated with dilating pupil as a result of dim lighting [2]. The attacks in our study were almost in the same situation. The examinations performing at the same timepoint was the most simple and effective way to reduce the confounding effect. Meanwhile, the previous studies about the diurnal variations of choroidal thickness (CT) found that the maximum at 0300 hours, and the minimum at 1800 hours, which meant the CT gradually expand after sunset until midnight [3]. We speculate the trend of choroid expanding might play a role in the angle closure.

Second, our research team has recently published several articles about the topographic variation of the choroid. We found that POAG/PACD eyes had a thicker anterior choroid than the controls. However, there was no significant difference in the posterior choroidal thickness among the groups. The anterior choroid might play a role in the pathogenesis of glaucoma [4]. Meanwhile we found that Valsalva manoeuvre did not affect the posterior choroid, but it did cause thickening of the anterior choroid and the ciliary body, both of which led to a larger anterior placement of the ciliary body and a narrowed anterior chamber. The anterior (but not the posterior) choroid could be related to IOP

Xiulan Zhang

zhangx12@mail.sysu.edu.cn

1 State Key Laboratory of Ophthalmology, Zhongshan Ophthalmic Center, Sun Yat-Sen University, Guangzhou 510060, China elevation and a narrowed anterior chamber in primary angle closure diseases $[5,6]$. The relationship between the anterior choroid and the blood pressure need further investigation.

Finally, we thank Cheong et al. for the kind comment.

Funding: This research was supported by the National Natural Science Foundation of China (81670847, 81600728). The Cultivation Projects for Young Teaching Staff at Sun Yat-sen University (17ykpy75).

\section{Compliance with ethical standards}

Conflict of interest The authors declare that they have no conflict of interest.

\section{References}

1. Cheong XK, Tan C. Comment on: Difference of uveal parameters between the acute primary angle closure eyes and the fellow eyes. Eye. 2018.

2. Pokhrel PK, Loftus SA. Ocular emergencies. Am Fam Physician. 2007;76:829-36.

3. Usui S, Ikuno Y, Akiba M, et al. Circadian changes in subfoveal choroidal thickness and the relationship with circulatory factors in healthy subjects. Invest Ophthalmol Vis Sci. 2012;53:2300-7.

4. Gao K, Li F, Li Y, et al. Anterior choroidal thickness increased in primary open-angle glaucoma and primary angle-closure disease eyes evidenced by ultrasound biomicroscopy and SS-OCT. Invest Ophthalmol Vis Sci. 2018;59:1270-7.

5. Li X, Wang W, Chen S, et al. Effects of valsalva maneuver on anterior chamber parameters and choroidal thickness in healthy Chinese: an AS-OCT and SS-OCT study. Invest Ophthalmol Vis Sci. 2016;57:T189-95.

6. Li F, Gao K, Li X, et al. Anterior but not posterior choroid changed before and during Valsalva manoeuvre in healthy Chinese: a UBM and SS-OCT study. Br J Ophthalmol. 2017;101:1714-19. 


\title{
Creating a virtual community of practice: an evaluation of ophthalmology-optometry Project ECHO
}

\author{
Michael Williams $\mathbb{D}^{1} \cdot$ Augusto Azuara Blanco ${ }^{2,3} \cdot{\text { Ruth } \mathrm{Hogg}^{2}}^{2}$ Gerry Mahon ${ }^{3} \cdot$ Margaret McMullan $^{4}$. \\ Raymond Curran ${ }^{4} \cdot$ Max Watson ${ }^{5}$
}

Received: 3 July 2018 / Accepted: 6 July 2018 / Published online: 1 August 2018

(c) The Royal College of Ophthalmologists 2018

As the absolute number of elderly people rises and with the advent of OCT scanners, community optometrists (COs) are seeing more abnormalities in their routine practice. Furthermore hospital eye services face a challenge keeping up with demand. Project ECHO (Extension for Community Healthcare Outcomes) is an educational model that partners primary healthcare providers with specialists, creating a 'community of practice'. ECHO has been applied to many areas of healthcare (http://echo. unm.edu accessed June 2018), but not previously to eye care. We describe the first ECHO project involving ophthalmologists (in the 'hub') and COs (in the 'spokes'). Some COs want enhanced roles: ECHO is a potential vehicle contributing to governance of shared eye care. The aims of this study were to assess the feasibility, acceptability and impact on knowledge and self-reported efficacy of the eye care ECHO and explore participants' impressions of it.

A mixed-methods prospective cohort study was performed on 12 ECHO sessions, held weekly. Each session had the same $21 \mathrm{COs}$, and in the hub one of two ophthalmologists who focused on macular disease or glaucoma. A talk was given by a hub-member, then COs presented cases. Due to the small sample size, descriptive statistics

Michael Williams

m.williams@qub.ac.uk

1 Centre for Medical Education, Queen's University of Belfast, Belfast, UK

2 Centre for Public Health, Queen's University of Belfast, Belfast, UK

3 Belfast Health and Social Care Trust, Belfast, UK

4 Northern Ireland Health and Social Care Board, Belfast, UK

5 Northern Ireland Hospice, Belfast, UK and non-parametric tests were used. Focus group data was analysed using thematic analysis.

Of the 21 spoke optometrists, $29 \%(6 / 21)$ had OCT scanners. In a knowledge assessment, marks improved from the pre-ECHO test (median 25/38, range 19-31), to the post-ECHO test (median 27/38, range 21-33) $(z=-2.3$, $p=0.019)$. Ninety-one percent (10/11) of COs who responded indicated they had learnt "a lot" through ECHO and 70\% (7/10) agreed that participating in ECHO had improved the care they provide for patients "a lot". Analysis of transcribed focus group discussions led to identification of three themes: professional and clinical learning occurring, expectations being exceeded, and suggestions for future ECHO sessions. An exemplar quotation was: “...certainly for me it will reduce some unnecessary referrals but it will also help me pick up on things that I really need to refer". Also 'diffusion of learning' occurred: "I found other colleagues asking me things and it was something that I could maybe bring as a question in ECHO, so the whole team did learn, and because I work in different practices, that was spread among different practices too."

Though numbers are small, the data suggest that ECHO was feasible, acceptable and could be an effective training modality. Focus groups discussions by participants in the ECHoES trial (unrelated to Project ECHO) revealed potential barriers to the deployment of optometrists in hospital-based ophthalmic care, including the need for "buy in" for optometric involvement [1]. 'Buy in' was suggested to depend on training delivered in a way that would reassure the ophthalmologist: ECHO may provide this.

As the ECHO model is replicable, the challenge would be to nurture local relationships formed to ensure sustainable participation, and eventually to incorporate ECHO's multidisciplinary networks permanently into service delivery in a way that is acceptable to all, safe and demonstrably benefits patients. 
Acknowledgements

Funding This ECHO project was supported by funding from the Northern Ireland Health and Social Care Board.

\section{Compliance with ethical standards}

Conflict of interest The authors declare that they have no conflict of interest.

\section{Reference}

1. Reeves BC, Scott LJ, Taylor J, Hogg R, Rogers CA, Wordsworth S, et al. The Effectiveness, cost-effectiveness and acceptability of Community versus Hospital Eye Service followup for patients with neovascular age-related macular degeneration with quiescent disease (ECHoES): a virtual randomised balanced incomplete block trial. Health Technol Assess. 2016;20: $1-120$.

\title{
Post-injection endophthalmitis in eyes receiving vs. not receiving topical antibiotic prophylaxis in Northern Thailand
}

\author{
Voraporn Chaikitmongkol $^{1} \cdot$ Onnisa Nanegrungsunk ${ }^{2} \cdot$ Direk Patikulsila $^{1} \cdot$ Janejit Choovuthayakorn ${ }^{1}$. \\ Nawat Watanachai ${ }^{1} \cdot$ Paradee Kunavisarut $^{1} \cdot$ Nimitr Ittipunkul $^{1} \cdot$ Neil M. Bressler $^{3}$
}

Received: 8 February 2018 / Revised: 26 April 2018 / Accepted: 7 May 2018 / Published online: 14 August 2018

(c) The Royal College of Ophthalmologists 2018

\section{Introduction}

Endophthalmitis following intravitreous anti-vascular endothelial growth factor (VEGF) injection is a rare sight-threatening condition. Several Western references suggest lower rates of post-injection endophthalmitis can be achieved without topical antibiotic prophylaxis [1-3]. However, a majority of physicians in the Asia-Pacific region still prescribe antibiotic prophylaxis [4], anecdotally stating concern that differences in environmental factors (e.g., tropical climates) or patient factors in the region might cause higher risk of endophthalmitis, comparing to the Western settings, to warrant this prophylaxis, despite little scientific rationale supporting such use. To our knowledge, little is known regarding incidence of post-injection endophthalmitis without antibiotic

Neil M. Bressler

nmboffice@jhmi.edu

1 Retina Division, Department of Ophthalmology, Faculty of Medicine, Chiang Mai University, Chiang Mai, Thailand

2 Department of Ophthalmology, Faculty of Medicine, Chiang Mai University, Chiang Mai, Thailand

3 Retina Division, Wilmer Eye Institute, Johns Hopkins University School of Medicine, Baltimore, MD, USA prophylaxis in a developing countries in Asia or elsewhere. Therefore, this study determined incidence of postinjection endophthalmitis with vs. without topical antibiotic prophylaxis at a university-based practice in Northern Thailand.

\section{Materials and methods}

IRB-approved prospective case series of patients receiving anti-VEGF injections at Chiang Mai University Hospital between May 2015 and September 2016 with follow-up anticipated for $\geq 3$ weeks after injections were recruited. Before injections, study eyes were examined by slit-lamp biomicroscopy, ensuring no intraocular inflammation. Endophthalmitis was evaluated at $4 \pm 1$ weeks after the injection, and defined as severe inflammation in both anterior chamber and vitreous cavity associated with pain, redness, or decreased vision, regardless of whether subsequent cultures were positive.

A standardized intravitreous anti-VEGF injection protocol was strictly applied, including use of sterile drape, sterile eyelid speculum, sterile glove, procedure mask, and application of povidone-iodine, twice, over eyelid and conjunctival sac, and over injection site using a povidone-iodine soaked cotton tip for $\geq 30 \mathrm{~s}$ before injection. 
Table 1 Demographics of participants and study eyes in each subgroup (receiving vs. not receiving antibiotics) and overall

\begin{tabular}{|c|c|c|c|}
\hline & \multicolumn{2}{|c|}{ Topical antibiotic prophylaxis } & \multirow{2}{*}{$\begin{array}{l}\text { Overall, } 1043 \\
\text { participants }(\%)\end{array}$} \\
\hline & $\begin{array}{l}\text { Receiving, } 381 \\
\text { participants (\%) }\end{array}$ & $\begin{array}{l}\text { Not receiving, } 662 \\
\text { participants }(\%)\end{array}$ & \\
\hline Age (mean $\pm \mathrm{SD}$, years $)$ & $61.6 \pm 7.8$ & $60.4 \pm 7.8$ & $60.8 \pm 7.8$ \\
\hline \multicolumn{4}{|l|}{ Gender } \\
\hline Men & $193(50.7)$ & $333(50.3)$ & $526(50.4)$ \\
\hline Women & $188(49.3)$ & $329(49.7)$ & 517 (49.6) \\
\hline \multicolumn{4}{|l|}{ Nationality } \\
\hline Thai & $377(99.0)$ & $656(99.1)$ & $1033(99.0)$ \\
\hline Hill-tribe & $4(1.0)$ & $6(0.9)$ & $10(1.0)$ \\
\hline \multicolumn{4}{|l|}{ Occupation } \\
\hline $\begin{array}{l}\text { Office workers/indoor } \\
\text { workers/retirees }\end{array}$ & $317(83.2)$ & $539(81.4)$ & $856(82.1)$ \\
\hline Outdoor workers $^{\mathrm{a}}$ & $64(16.8)$ & $123(18.6)$ & 187 (17.9) \\
\hline \multicolumn{4}{|l|}{ Educational level } \\
\hline Primary school or lower & $219(57.5)$ & $408(61.6)$ & $627(60.1)$ \\
\hline $\begin{array}{l}\text { Secondary school or } \\
\text { higher }\end{array}$ & $162(42.5)$ & $254(38.4)$ & $416(39.9)$ \\
\hline \multicolumn{4}{|l|}{ Study eye } \\
\hline Unilateral & $346(90.8)$ & $565(85.3)$ & $911(87.3)$ \\
\hline \multirow[t]{2}{*}{ Bilateral } & $35(9.2)$ & $97(14.7)$ & $132(12.7)$ \\
\hline & $\begin{array}{l}\text { Receiving, } 1407 \\
\text { injections }(\%)\end{array}$ & $\begin{array}{l}\text { Not receiving, } 2856 \\
\text { injections }(\%)\end{array}$ & $\begin{array}{l}\text { Overall, } 4263 \\
\text { injections }(\%)\end{array}$ \\
\hline \multicolumn{4}{|l|}{ Indication for injection ${ }^{\mathrm{b}}$} \\
\hline DME & 445 (31.6) & $1124(39.4)$ & $1569(36.8)$ \\
\hline PCV & $206(14.6)$ & $574(20.1)$ & $780(18.3)$ \\
\hline NV AMD & $303(21.5)$ & $371(13.0)$ & $674(15.8)$ \\
\hline RVO with ME & $253(18.0)$ & $441(15.4)$ & $694(16.3)$ \\
\hline $\begin{array}{l}\text { PDR-related condition e.g., } \\
\text { VH }\end{array}$ & $138(9.8)$ & $220(7.7)$ & $358(8.4)$ \\
\hline Others & $62(4.4)$ & $126(4.4)$ & $188(4.4)$ \\
\hline \multicolumn{4}{|l|}{ Anti-VEGF agents ${ }^{c}$} \\
\hline Aflibercept & $216(15.4)$ & $758(26.5)$ & $974(22.8)$ \\
\hline Bevacizumab & $1104(78.5)$ & 2011(70.4) & $3115(73.1)$ \\
\hline Ranibizumab & $87(6.2)$ & $87(3.0)$ & $174(4.1)$ \\
\hline
\end{tabular}

${ }^{a}$ For example, agricultural workers, construction workers

${ }^{\mathrm{b}} \mathrm{DME}$ diabetic macular edema, PCV polypoidal choroidal vasculopathy, NV AMD neovascular age-related macular degeneration, RVO retinal vein occlusion, ME macular edema, PDR proliferative diabetic retinopathy, VH vitreous hemorrhage, anti-VEGF anti-vascular endothelial growth factor

${ }^{c}$ Bevacizumab was re-packaged in sterile fashion by the hospital pharmacy department into multiple doses of $1.25 \mathrm{mg} / 0.05 \mathrm{ml}$. Each dose was stored in a sterile insulin syringe, while ranibizumab $(0.5 \mathrm{mg} / 0.05 \mathrm{ml})$ and aflibercept $(2.0 \mathrm{mg} / 0.05 \mathrm{ml})$ were prepared by physicians at the time of each injection
Subsequently, participants received or did not receive topical antibiotics depending on their physicians' preferences. Three of six retina specialists (DP, PK, NI) always prescribed antibiotics $\left(\right.$ Tobramycin $^{\circledR}$ ) before or after injections for 5-7 days; the other three (JC, $\mathrm{NW}, \mathrm{VC}$ ) did not prescribe any antibiotics as part of standard care.

\section{Results}

Of 4263 injections (1043 participants) meeting eligibility criteria, 1407 (33\%) received antibiotic prophylaxis, and $2856(67 \%)$ did not receive any antibiotics. Demographics are shown in Table 1. There was one case of culture-negative post-injection endophthalmitis, which subsequently underwent vitrectomy, among eyes 
Table 2 Incidence of endophthalmitis in eyes receiving vs. not receiving antibiotic prophylaxis

\begin{tabular}{lllll}
\hline & $\begin{array}{l}\text { Topical antibiotic } \\
\text { prophylaxis }\end{array}$ & $\begin{array}{l}\text { Difference of incidence }(95 \% \\
\text { confidential interval) }\end{array}$ & P value \\
\cline { 2 - 3 } & Receiving $^{\mathrm{a}}$ & Not receiving & \\
\hline $\begin{array}{l}\text { Number of intravitreous } \\
\text { injections }\end{array}$ & 1407 & 2856 & & \\
\begin{tabular}{l} 
Incidence of endophthalmitis \\
\hline
\end{tabular} & $1(0.07 \%)$ & $-(0 \%)$ & $0.07 \%(-0.12 \%$ to $0.26 \%)$ & 0.72 \\
\hline
\end{tabular}

Tobramycin eye drops for 5-7 days before or after intravitreous injection or both before and after injection receiving antibiotics (1/1407 injections; 0.07\%). No endophthalmitis occurred among eyes not receiving antibiotics $(0 / 2856$ injections; $0 \%)$ for a difference of $0.07 \%$ ( $95 \%$ confidence interval: -0.12 to $0.26 \%$, $P=0.728$; Table 2).

\section{Discussion}

To our knowledge, this study is among the first to explore an incidence of post-injection endophthalmitis when no antibiotic prophylaxis was given in a developing-country setting. The setting, a government hospital which mainly serves lower socioeconomic patients within geographically tropical climates, may be a good representative for individuals believed by some to be at higher risk for post-injection endophthalmitis in developing countries. The results, though not a randomized clinical trial, did not reveal an increased incidence of endophthalmitis in eyes not receiving antibiotic prophylaxis, similar to Western reports [1-3]. These data support continued use of povidone-iodine for $\geq 30 \mathrm{~s}$ prior to intravitreous injection [5] but not topical antibiotics for prophylaxis against post-injection endophthalmitis.

Funding Funding of this study was provided by Research Committee, Faculty of Medicine, Chiang Mai University, and unrestricted donations to Johns Hopkins University for retina research. The sponsor had no role in the design or conduct of this research.

\section{Compliance with ethical standards}

Conflict of interest All authors have completed and submitted the ICMJE Form for Disclosure of Potential Conflicts of Interest. VC reported receiving research grant from Bayer and ThromboGenics; and receiving honoraria and travel reimbursement from Allergan and Novartis. ON reported no financial disclosure. DP reported working as a consultant, receiving honoraria and travel reimbursement from Bayer and Novartis; and receiving honoraria and travel reimbursement from Alcon. JC and NW reported receiving honoraria and travel reimbursement from Alcon, Allergan, Bayer and Novartis. PK reported receiving honoraria and travel reimbursement from Novartis. NI reported no financial disclosure. NMB reported receiving grants to employer, Johns Hopkins University; and from Bayer, Novartis, Roche (Genentech) and Samsung. No other disclosures were reported.

\section{References}

1. Bhavsar AR, Stockdale CR, Ferris FL 3rd, Brucker AJ, Bressler NM, Glassman AR. Diabetic Retinopathy Clinical Research Network. Update on risk of endophthalmitis after intravitreal drug injections and potential impact of elimination of topical antibiotics. Arch Ophthalmol. 2012;130:809-10.

2. Storey P, Dollin M, Pitcher J, Reddy S, Vojtko J, Vander J, et al. Post-Injection Endophthalmitis Study Team. The role of topical antibiotic prophylaxis to prevent endophthalmitis after intravitreal injection. Ophthalmology. 2014;121:283-9.

3. Meredith TA, McCannel CA, Barr C, Doft BH, Peskin E, Maguire MG, Comparison of Age-Related Macular Degeneration Treatments Trials Research Group. et al. Postinjection endophthalmitis in the comparison of age-related macular degeneration treatments trials (CATT). Ophthalmology. 2015;122:817-21.

4. American Society of Retinal Specialists. 2014 global trends in retina. 2014. https://www.asrs.org/content/documents/2014_globa 1_trends_comprehensivepostmtg.pdf. Accessed 20 April 2018.

5. Bhavsar AR, Glassman AR, Stockdale CR, Jampol LM. Diabetic Retinopathy Clinical Research Network. Elimination of topical antibiotics for intravitreous injections and the importance of using povidone-iodine: update from the Diabetic Retinopathy Clinical Research Network. JAMA Ophthalmol. 2016;134:1181-3. 


\title{
A novel and effective ophthalmology morbidity and mortality meeting
}

\author{
K. L. Chan ${ }^{1} \cdot$ J. Deutsch ${ }^{1} \cdot$ G. Morphis ${ }^{1} \cdot$ S. Scotcher ${ }^{1} \cdot$ A. Smith ${ }^{1} \cdot$ B. While ${ }^{1} \cdot$ S. Madge ${ }^{1}$ \\ Received: 9 August 2018 / Accepted: 10 August 2018 / Published online: 7 September 2018 \\ (c) The Royal College of Ophthalmologists 2018
}

Morbidity and mortality (M\&M) meetings drive learning from clinical outcomes and improve service delivery in surgery [1]. They are regular scheduled meetings that discuss adverse surgical outcomes for patients and were originally designed for surgical training. M\&M meetings are common place in other surgical specialties and are recommended by the Royal College of Surgeons as part of maintaining and improving patient safety [2]. However, M\&M practice appears to be lacking in UK ophthalmology with no recommendations from the Royal College of Ophthalmologists or research into its potential benefits. We present a novel method of integrating M\&M meetings to ophthalmology practice which has been running in our department since November 2016. We also present a qualitative analysis of its impact on the department since its inception.

To collect M\&M incidents, we took a simple twopronged approach: a 'glitchbook' was placed in the ophthalmic theatre to prospectively capture at source any surgical complications; in addition, an email address was created to capture $M \& M$ in the clinic setting. In both methods, incidents are reported by the surgeon and/or any auxiliary member of the team.

The M\&M meeting was integrated into the departments by two monthly audit meetings to reduce the administrative burden of a separate M\&M meeting. These meetings included the wider multidisciplinary team: consultants, trainees, nursing staff and auxiliary staff. Each incident collected is presented by the operating surgeon, which can be in the form of a short description of events, through to a full presentation with a video of the operation. Following the presentations of the case, an open and

Senthil Maharajan

senthil.maharajan@nuh.nhs.uk

1 Victoria Eye Unit, Hereford County Hospital, Hereford HR1 2ER Herefordshire, UK reflective discussion about the surgical issues occur with involvement of the whole team. In each case, learning points and reflections are noted and any changes needed are implemented and discussed at the next meeting.

A qualitative analysis involving semi-structured interviews and questionnaires was performed to investigate the impact of M\&M meetings on the department. There were multiple themes that arose from this study: training and education, cultural change and improving patient care. All surgeons from a trainee to a consultant felt that this was an excellent opportunity and resource for learning. The trainees particularly felt the benefit from experienced feedback from multiple consultants and the chance to hone their presentation skills. All members of the staff felt that the M\&M meetings had fostered a blame-free and open culture within the department. The combination of discussing M\&M issues and open culture was felt to be strongly driving improvements in patient safety.

Our departments experience of M\&M meetings is that we have found it an easy process to implement, with great benefits for training and patient safety. We recommend that UK ophthalmology keeps up with the other specialties and adopts $\mathrm{M} \& \mathrm{M}$ as a standard practice.

\section{Compliance with ethical standards}

Conflict of interest The authors declare that they have no conflict of interest.

\section{References}

1. Good Surgical Practice-Royal College of Surgeons. https://www. rcseng.ac.uk/standards-and-research/gsp.

2. Morbidity and Mortality Meetings-Royal College of Surgeons. https://www.rcseng.ac.uk/standards-and-research/standards-andguidance/good-practice-guides/morbidity-and-mortality-meetings 


\title{
Health tourism and the need for occasional strong paternalism: complications and management of cosmetic anterior chamber iris implantation
}

\author{
Craig Wilde ${ }^{1} \cdot$ Andrew R. Ross ${ }^{1} \cdot$ Senthil Maharajan ${ }^{1}$
}

Received: 16 July 2018 / Accepted: 7 August 2018 / Published online: 31 August 2018

(c) The Royal College of Ophthalmologists 2018

Health tourism involves intentional travel abroad to obtain private medical care. Motivations are numerous, ranging from financial (affordable treatment) to accessing care unavailable at home [1]. Evidence regarding risk is often limited, sometimes speculative, with little information to guide decisions. It can be biased, based on sensationalist reports, often produced by people with inadequate expertise in such procedures [2]. Most reports reflect individual cases or small case series of problems. Limited studies publishing complication rates for health tourism exist [3], making risk hard to assess. Complications may be underreported where surgery is associated with financial gain and where patients travel from abroad with poor follow up. A survey by the British Association of Plastic, Reconstructive and Aesthetic Surgery found $37 \%$ of members had seen complications of health tourism [4], raising concerns for an individual's risk but the burden within public healthcare facilities treating complications.

Several publications exist on the dangers of cosmetic iris implants [5], the risks of which have been known for years. We report a case of devastating complications following the procedure.

A 45-year old phakic male had bilateral BrightOcular implantation in 2013 in Jordan (Fig. 1a). Upon return he periodically developed red, painful, photophobic eyes. Intraocular pressures were elevated during those episodes with associated anterior chamber inflammation. Gonioscopy showed peripheral iris entrapment into the iridocorneal angle (Fig. 1b). Uveitis, glaucoma, hypheama syndrome

Senthil Maharajan

senthil.maharajan@nuh.nhs.uk

1 Ophthalmology and Vision Sciences, Division of Clinical Neurosciences, B Floor, EENT Centre, Queen's Medical Centre, University of Nottingham, Nottingham, UK was diagnosed. He was asked to return promptly to Jordon for implant removal. After a period of delay, he went as advised. On return, subsequent examination revealed bilateral corectopia, extensive iris atrophy, stretch holes, peripheral anterior synechiae and cataract formation (Fig. 1c, d). Intraocular pressures were elevated and cyclodiode was performed. Despite implant removal, progressive endothelial cell loss with corneal decompensation ensued (Fig. 1e). The patient was listed for Descemets stripping automated endothelial keratoplasty (DSAEK). While awaiting a graft he developed bacterial keratitis (Fig. 1f), resulting in right eye corneal scarring, and the need for penetrating keratoplasty (PK) bilaterally. The right eye did well (Fig. 1g), with the graft remaining clear and Snellen best corrected visual acuity (BCVA) of 6/6. The left eye vision recovered, but developed a suture abscess with subsequent graft failure (Fig. 1h). The patient is awaiting a second PK (with BCVA of hand movements).

Despite previous reports of significant complications, cosmetic iris implants are still advertised across multiple websites and social media platforms in various countries. Some testify the procedure as safe with no patient going blind. We highlight the dangers, reporting potential for bilateral pain, severe sight loss and morbidity. We emphasise visual rehabilitation is not always simple following implant extraction. Cataracts, glaucoma surgery, iris deformation and endothelial cell loss constitute multiple challenges. We join colleagues in calling for international eye surgery communities to increase awareness of this potentially sight threatening issue and informing patients of risks prior to undertaking surgery. Professional governing bodies should encourage reporting of complications from such cosmetic surgery and consider closer monitoring. Better international legislation restricting dangerous surgical practice could be explored. 

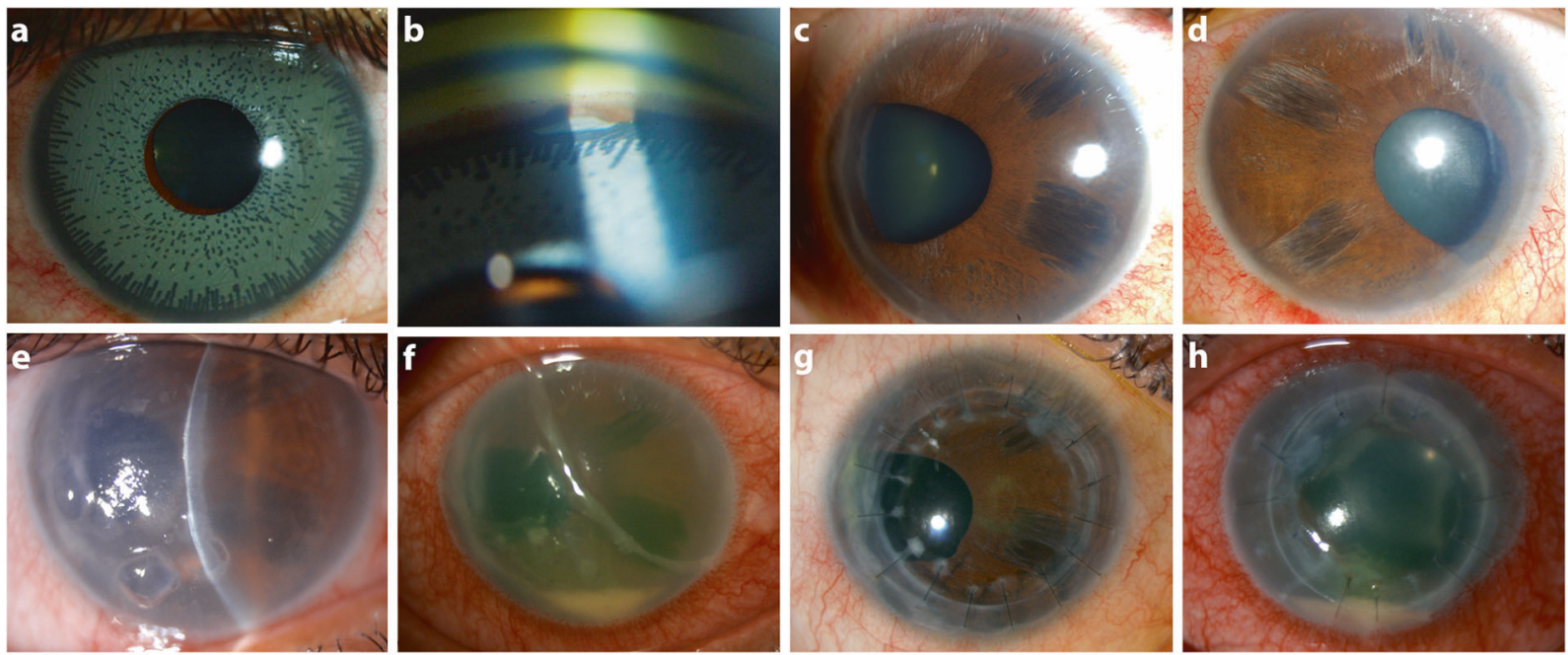

Fig. 1 a BrightOcular single piece silicone iris implant in situ with conjunctival injection secondary to uveitis. The implant appears decentred in relation to the pupil, reflecting the issue of poor fit of a single size implant. Whether the pigment used to colour the implant is inert over time or contributes to inflammation or endothelial toxicity and cell loss remains to be elucidated. b Gonioscopy photograph showing the iridocorneal angle with the BrightOcular in situ. One of several foot processes is impinging the peripheral iris and is in part responsible for stretch hole formation. Secondary glaucoma can develop from pigment dispersion from contact between the implant and iris, direct trabecular meshwork damage from implant edge and chronic inflammation with angle closure. Whether iris and implant were adherent and if trauma occurred during implant removal is unknown. Significant adhesions have been reported. c, d Eyes following BrightOcular removal. Temporal corneal wounds are visible from surgery to explant the artificial iris. Conjunctival injection secondary to persistent anterior uveitis with development of early cataracts secondary to either direct contact from the implants, associated inflammation or topical steroid use. Widespread permanent iris damage is evident. Symmetrical temporal corectopia is visible, with iris thinning in the opposite nasal segment with large corresponding stretch holes. e Corneal decompensation with multiple large epithelial bullae and associated epithelial defects. The patient suffers significant episodes of pain. f Acutely painful red eye secondary to Pseudomonas Aeruginosa keratitis. g Clear PK in right eye with patient achieving good BCVA. h Failed corneal graft in left eye. Inferior suture abscess and hypopyon. There is significant capsular opacification

\section{Compliance with ethical standards}

Conflict of interest The authors declare that they have no conflict of interest.

\section{References}

1. Johnston R, Crooks VA, Snyder J. "I didn't even know what I was looking for": a qualitative study of the decision-making processes of Canadian medical tourists. Glob Health. 2012;8:23.
2. Crooks VA, Turner L, Cohen IG, Bristeir J, Snyder J, Casey V, et al. Ethical and legal implications of the risks of medical tourism for patients: a qualitative study of Canadian health and safety representatives' perspectives. BMJ Open. 2013;3:pii: e002302.

3. Al-Hinai SS, Al-Busaidi AS, Al-Busaidi IH. Medical tourism abroad: a new challenge to Oman's health system - Al Dakhilya region experience. Sultan Qaboos Univ Med J. 2011;11:477-84.

4. Jeevan R, Armstrong A. Cosmetic tourism and the burden on the NHS. J Plast Reconstr Aesthet Surg. 2008;61:1423-4.

5. Hoguet A, Ritterband D, Koplin R, Wu E, Raviv T, Aljian J, et al. Serious ocular complications of cosmetic iris implants in 14 eyes. J Cataract Refract Surg. 2012;38:387-93. 